\title{
Model-Based Estimation of Metal Analysis in Steel Converters
}

\author{
Andreas Johansson ${ }^{12}$, Alexander Medvedev ${ }^{2}$, and Daniel Widlund ${ }^{3}$
}

\begin{abstract}
A nonlinear observer is developed for metal analysis in the top blown converter process. It is shown that the observer is asymptotically stable and a region of attraction, where the estimation error converges, is derived. Using real plant data from the converter at SSAB Oxelösund $\mathrm{AB}$, the observer is shown to provide accurate estimates of the carbon content.
\end{abstract}

\section{Introduction}

The top blown basic oxygen method was developed 50 years ago and is today the predominant process for steelmaking. In 1995 it accounted for nearly $60 \%$ of the world total crude steel production of almost 750 million metric tons.

The aim of the process is to reduce the contents of carbon and silicon and other components in the hot metal from the blast furnace. The main quality measure of the final product is the carbon content. On the one hand, if the preset upper limit is exceeded, then the process must be repeated, which is very time consuming.

On the other hand, there is a considerable cost in terms of consumption of resources, such as oxygen, energy, and production time, when the final carbon content is made unnecessarily low.

Being able to accurately estimate the metal analysis, especially the content of carbon, is thus important to ensure the quality of the steel, minimize the consumption of resources and to avoid re-blowing. The use of an analytical process model is a promising way of achieving this.

There exist a few commercial systems for on-line estimation of metal analysis, e.g. MEFCON [1], developed by the Foundation for Metallurgical Research, MEFOS in Luleå, Sweden. MEFCON has a carbon content hitting rate of $95 \%$ in a window of $\pm 0.007-0.012 \%$ depending on the final carbon content.

To obtain higher estimation accuracy and robustness against modeling errors, it is necessary to utilize mul-

\footnotetext{
${ }^{1}$ e-Mail Andreas.Johansson@sm.luth.se Phone +46920723 $34 \mathrm{Fax}+4692091558$

${ }^{2}$ Luleå University of Technology, Control Engineering Group, SE-971 87 Luleå, Sweden

${ }^{3}$ SSAB Oxelösund AB, SE-613 80 Oxelösund, Sweden
}

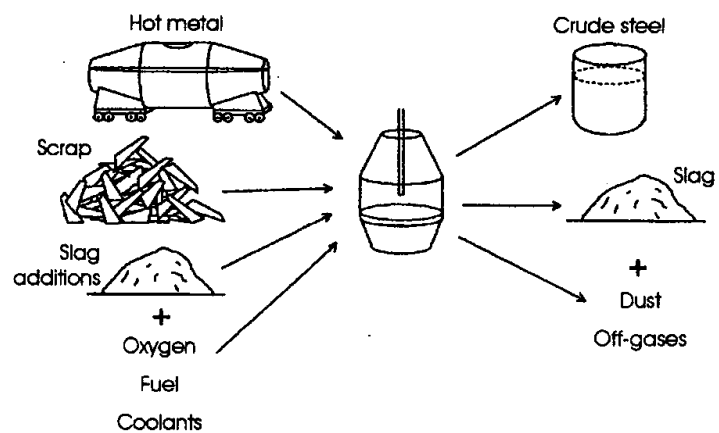

Figure 1: The top blown converter process

tiple information sources, i.e. both a model and the relevant measurements. This calls for feedback of the estimation error, i.e. constructing an observer. Since the process is highly nonlinear and has direct coupling between input and and output, this is a nontrivial task.

Observers for nonlinear systems are for example treated in [2]. A number of methods exist, e.g. the extended Kalman filter and different linearization techniques. Each of these methods have their own advantages but also inherent drawbacks such as computational complexity and lack of stability guarantee. An exhaustive treatment of nonlinear systems regarding stability and other properties is [3].

\section{Process description}

Fig. 1 is a schematic drawing of the converter process with its raw materials and products.

The operation is started by charging hot metal, scrap, and slag forming agents into the converter. Other additives, such as fuel, in the form of ferrosilicon or coke, and cooling agents, in the form of iron oxides, can be added throughout the blow.

Oxygen $\left(\mathrm{O}_{2}\right)$ is blown at a supersonic rate onto the metal surface and oxidises the metal components, mainly iron ( $\mathrm{Fe}$ ), silicon ( $\mathrm{Si}$ ), manganese (Mn), and carbon (C). The oxides, together with metal droplets, form a foaming slag, in which more carbon will react with the oxides and produce carbon monoxide (CO). In combination with oxygen, some of the carbon monoxide will produce carbon dioxide $\left(\mathrm{CO}_{2}\right)$. 
The converter is controlled by an operator, who judges the state of the process based upon a number of measurements, e.g. a sound level measurement obtained by a sonicmeter and analysis of the off-gas [4]. The sound level gives information of the thickness of the foaming slag, while the off-gas analysis provides information on the decarburization rate, i.e. the rate at which carbon is oxidised.

The process takes approximately 15-20 minutes and is stopped by the operator when the content of carbon in the metal is considered right, based upon the available measurements. The quality of the final product is therefore highly dependent on the experience and the judgement of the individual operator.

\section{Process model of the steel converter}

A process model has been developed at the Division of Metallurgy of the Royal Institute of Technology in Stockholm. It can be simplified to

$$
\begin{aligned}
\dot{p} & =A p+B w+E y \\
y & =h(p, u)
\end{aligned}
$$

where the state vector $p=\left[\begin{array}{ll}p_{\mathrm{Si}} & p_{\mathrm{C}}\end{array}\right]^{T}$ represents the total contents of $\mathrm{C}$ and $\mathrm{Si}$ in the metal, given in moles. The linear part of (1) is asymptotically stable since $A=\operatorname{diag}\left(-a_{1},-a_{2}\right)$ where the coefficients $a_{1}$ and $a_{2}$ are both positive.

The output $y$ denotes the decarburization rate and is a nonlinear function of the state $p$ and the input $y$ (see Appendix A). Since it represents the rate at which carbon is oxidized, it affects the derivative of the carbon content through the coefficient -1 , which motivates the vector $E=\left[\begin{array}{ll}0 & -1\end{array}\right]^{T}$.

\subsection{Input signals}

Inflow of oxygen and the off-gas flow is represented by $u$. The contribution of $\mathrm{C}$ and $\mathrm{Si}$ from scrap and other additives constitute the vector $w$. It is assumed that the additives dissolve at a constant rate and thus the elements of $w$ have the form

$$
w_{i}(t)= \begin{cases}m_{i} / T_{i} & t_{i}+\tau_{i}<t<t_{i}+\tau_{i}+T_{i} \\ 0 & \text { Otherwise }\end{cases}
$$

where $t_{i}$ is the time at which the amount $m_{i}$ of $\mathrm{C}$ or $\mathrm{Si}$ from additive $i$ is added. The time it takes to heat the additive to the melting point is denoted $\tau_{i}$ and $T_{i}$ is the time it takes for the additive to completely dissolve. The matrix $B$ consists of two rows of zeros and ones which determines whether the corresponding additive contributes to the amount of $\mathrm{C}$ or Si.

The time $t_{i}$ is completely known, but $\tau_{i}$ and $T_{i}$ as well as $m_{i}$ are parameters that account for the main source of uncertainty in the model.

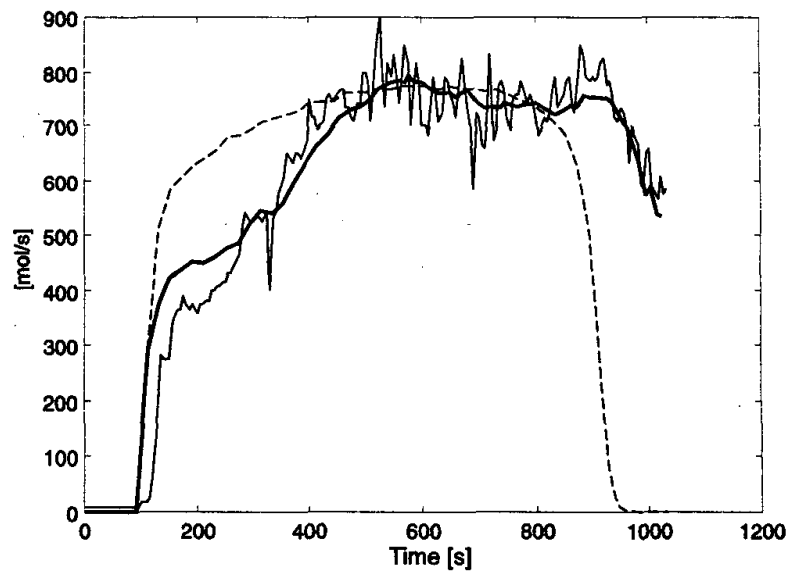

Figure 2: Estimated decarburization rate. Open loop (dashed line), observer (thick solid line) and offgas analysis (thin solid line)

\subsection{Output signals}

The decarburization rate $y$ which is indicated as output signal in (1) is not an actual measurement. Instead, the off-gas flow and amounts of $\mathrm{CO}, \mathrm{CO}_{2}$, and $\mathrm{O}_{2}$ are measured, represented by $f_{\mathrm{og}}$ and the vector $\psi$, respectively. The model of the relation between $y, f_{\text {og }}$, and $\psi$ can be found in Appendix A.

Since the three components in the off-gas analysis depend only on the decarburization rate, they can be regarded as redundant measurements of the latter. An estimate of the decarburization rate is thus calculated by the control system of the plant and this estimate is used as a measured output.

There is also a time delay in the measurement of $\psi$ due to the time it takes to transport the gas to the analysis equipment. This time delay is, however, neglected in the analysis, since the results do not seem to be affected by taking it into account.

\subsection{Model validation}

Fig. 2 (dashed line) shows a simulation of the decarburization rate using (1) with real input data from the plant. Comparing it to the decarburization rate estimated from the off-gas analysis (thin, solid line) shows that although the model overestimates the decarburization rate during the initial period (100 to 400 seconds), which results in a too early drop at the end of the blow, the levels agree fairly, and therefore the model is accepted.

A comparison between the simulated carbon content (Fig. 3, dashed line) and the measured final value (cross), however, indicates that the open-loop model does not produce sufficiently accurate estimates of the carbon content. Feedback of the measured signals should therefore be applied to improve the results. 


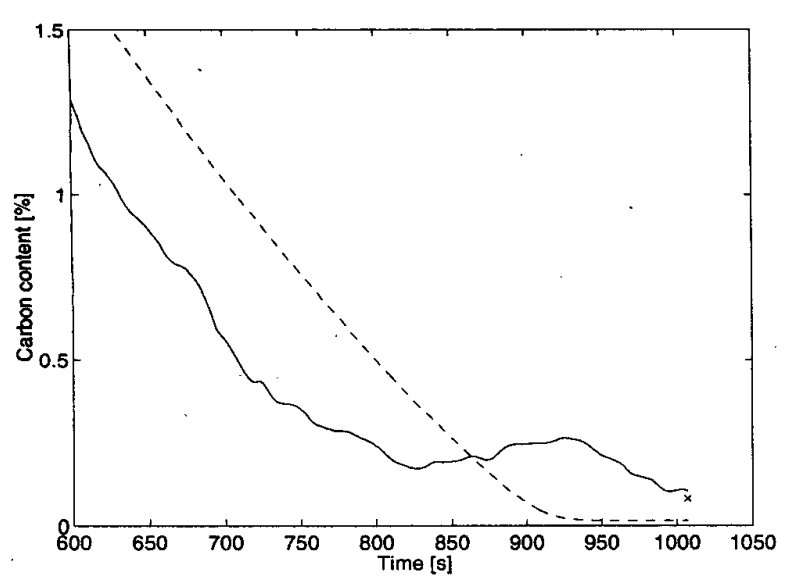

Figure 3: Estimate of carbon content using no feedback (dashed line) and linear feedback (solid line). Cross marks measured final value

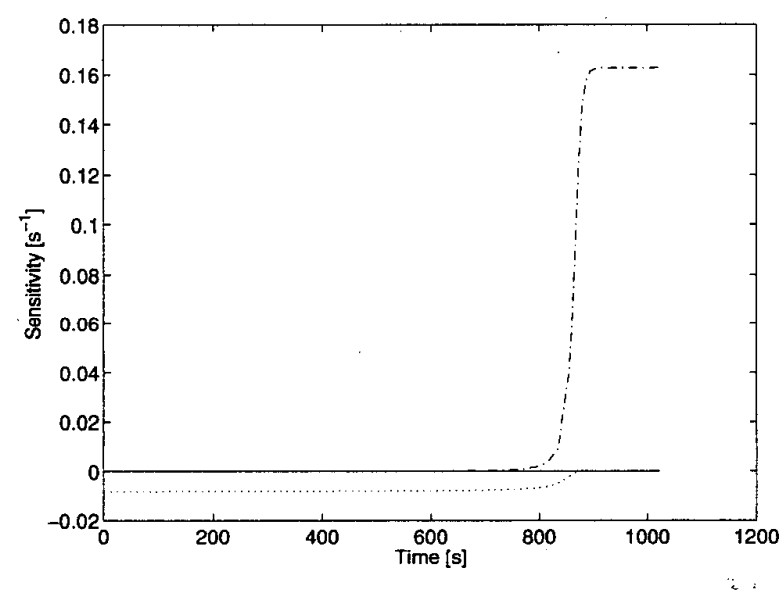

Figure 4: Sensitivity of the output to the silicon content (dotted) and to the carbon content (dashdotted) through an entire blow

\section{State estimation}

Designing a feedback from all three components of the off-gas analysis $\psi$ to an estimate $\hat{p}$ of the state vector $p$ is a complicated task, which is the reason for feeding back the estimated decarburization rate $y$ instead. This signal is scalar and likely to contain the major part of the relevant information in the off-gas analysis.

A linear feedback can be adjusted so that the estimated carbon content converges to the measured final value (Fig. 3, solid line). This is however accomplished at the price of unreliable estimates during the rest of the blowing period. The failure of the linear feedback can be explained with the sensitivity of the output with respect to the state vector, i.e.

$$
h^{\prime}(p, u) \triangleq \frac{\partial h(p, u)}{\partial p}
$$

The lower part of Fig. 4 shows the sensitivity to the silicon content (dotted) and to the carbon content (dashdotted). It can be seen that the output is sensitive to the carbon content only during the last part of the blowing period. Intuitively, there is no point in feeding back the output error to influence the estimate of a certain state variable when the output is insensitive to that state variable.

An observer is therefore proposed where the output error is weighted by the sensitivity of the output with respect to the state $i . e$.

$$
\begin{aligned}
& \dot{\hat{p}}=A \hat{p}+B w+E y+k h^{\prime}(\hat{p}, u)(y-\hat{y}) \\
& \hat{y}=h(\hat{p}, u)
\end{aligned}
$$

The gain $k$ is chosen to be a scalar, which appears to give satisfying results for this second-order system. It can be shown, however, that the stability analysis in section 5 , with only minor modifications, would work for a diagonal gain.

The suggested observer could be compared to the Extended Kalman Filter where the output error is fed back using the gain $K(t)=P(t) h^{\prime}(\hat{p}, u) R^{-1}(t)$, where $R(t)$ represents the covariance of the measurement noise and $P(t)$ is an approximation of the covariance of the estimation error. The latter is obtained by solving a timevarying Riccati equation, which leads to considerable computational complexity and makes it difficult to verify the stability of the observer.

Analogies can also be made to the MIT rule for updating parameters of an adaptive control system [5]. The parameters $\theta$ are changed along the negative gradient of a quadratic loss function which leads to

$$
\dot{\theta}=-\gamma e \frac{\partial e}{\partial \theta}
$$

where $e$ is the output error and $\gamma$ is a weighting factor. Identifying $\hat{p}$ as the parameter vector and $y-\hat{y}$ as the output error leads to

$$
\dot{\hat{p}}=-\gamma(y-\hat{y}) \frac{\partial}{\partial \hat{p}}(y-\hat{y})=\gamma h^{\prime}(\hat{p}, u)(y-\hat{y})
$$

which is the feedback term of (2). The additional terms on the right hand side of (2) account for the fact that it deals with dynamical states rather than constant parameters, which is the case of the MIT rule.

\section{Stability analysis}

Defining the estimation error $\tilde{p} \triangleq p-\hat{p}$ and using (1) and (2) to calculate $\dot{\tilde{p}}$ yields the estimation error process

$$
\dot{\tilde{p}}=A \tilde{p}-k h^{\prime}(p-\tilde{p}, u)(h(p, u)-h(p-\tilde{p}, u))
$$


According to the mean value theorem there exists a $\xi \in[0,1]$ such that $\bar{p}=p-\xi \tilde{p}$ satisfies

$$
h(p, u)-h(p-\tilde{p}, u)=h^{\prime T}(\bar{p}, u) \tilde{p}
$$

If $p$ and $u$ are regarded as functions of time $t$, then it is possible to express $h^{\prime}(\hat{p}, u)$ and $h^{\prime}(\bar{p}, u)$ as functions of $\tilde{p}$ and $t$ according to

$$
\begin{aligned}
\hat{h}^{\prime}(\tilde{p}, t) & \triangleq\left[\begin{array}{ll}
\hat{h}_{\mathrm{Si}}^{\prime} & \hat{h}_{\mathrm{C}}^{\prime}
\end{array}\right]^{T} \\
& \triangleq h^{\prime}(\hat{p}, u)=h^{\prime}(p(t)-\tilde{p}, u(t)) \\
\bar{h}^{\prime}(\tilde{p}, t) & \triangleq\left[\begin{array}{ll}
\bar{h}_{\mathrm{Si}}^{\prime} & \bar{h}_{\mathrm{C}}^{\prime}
\end{array}\right]^{T} \\
& \triangleq h^{\prime}(\bar{p}, u)=h^{\prime}(p(t)-\xi \tilde{p}, u(t))
\end{aligned}
$$

The error equation can then be expressed as a nonlinear time-varying system

$$
\dot{\tilde{p}}=\Phi \tilde{p}
$$

where $\Phi=\Phi(\tilde{p}, t)=A-k \hat{h}^{\prime}(\tilde{p}, t) \bar{h}^{\prime}(\tilde{p}, t)^{T}$.

Proposition 1 Let $\mathbb{B}_{r}$ be the open ball of radius $r$ and $\Lambda(x, t)$ the largest eigenvalue of $F(x, t)+F(x, t)^{T}$. If $\Lambda(x, t) \leq \Lambda_{0}<0$ on $\mathbb{B}_{r} \times[0, \infty)$, then the nonlinear time-varying system

$$
\dot{x}=F(x, t) x
$$

is asymptotically stable and $\mathbb{B}_{r}$ is a region of attraction for $x=0$.

Proof. Omitted.

The eigenvalues $\lambda$ of $\Phi+\Phi^{T}$ are given by the roots of the equation $\operatorname{det}\left(\lambda I-\left(\Phi+\Phi^{T}\right)\right)=0$. Straightforward calculations yield

$$
\operatorname{det}\left(\lambda I-\left(\Phi+\Phi^{T}\right)\right)=\lambda^{2}+d(\tilde{p}, t) \lambda+n(\tilde{p}, t)
$$

where

$$
\begin{aligned}
d(\tilde{p}, t)= & 2 k\left(\hat{h}_{\mathrm{Si}}^{\prime} \bar{h}_{\mathrm{Si}}^{\prime}+\hat{h}_{\mathrm{C}}^{\prime} \bar{h}_{\mathrm{C}}^{\prime}\right)+2\left(a_{1}+a_{2}\right) \\
n(\tilde{p}, t)= & 4 a_{1} a_{2}+4 k\left(a_{2} \hat{h}_{\mathrm{Si}}^{\prime} \bar{h}_{\mathrm{Si}}^{\prime}+a_{1} \hat{h}_{\mathrm{C}}^{\prime} \bar{h}_{\mathrm{C}}^{\prime}\right) \\
& -k^{2}\left(\hat{h}_{\mathrm{Si}}^{\prime} \bar{h}_{\mathrm{C}}^{\prime}-\hat{h}_{\mathrm{C}}^{\prime} \bar{h}_{\mathrm{Si}}^{\prime}\right)^{2}
\end{aligned}
$$

Lemma 1 If both roots of the second degree polynomial $x^{2}+a x+b$ are real and negative then they satisfy $x<$ $-b / a$

\section{Proof. Omitted.}

The eigenvalues of $\Phi+\Phi^{T}$ must be real since it is symmetric and thus, according to Lemma 1 , the conditions $d(\tilde{p}, t)>0$ and $n(\tilde{p}, t)>0$ guarantee that both eigenvalues are negative and satisfy $\lambda<-n(\tilde{p}, t) / d(\tilde{p}, t)$. Since $a_{1}, a_{2}, \hat{h}_{\mathrm{C}}^{\prime}, \tilde{h}_{\mathrm{C}}^{\prime} \geq 0$ and $\hat{h}_{\mathrm{Si}}^{\prime}, \bar{h}_{\mathrm{Si}}^{\prime} \leq 0, d(\tilde{p}, t)$ is always positive and the only negative term in $n(\tilde{p}, t)$ is the third one, which should therefore be limited.
Lemma 2 Let $f$ and $g$ be differentiable functions on $\mathbb{B}_{R}$ and let $0 \leq \zeta \leq 1$. Then

$$
\begin{aligned}
& |f(x) g(\zeta x)-f(\zeta x) g(x)| \leq \\
& R \sup _{x \in \mathbb{B}_{R}}|g(x)| \sup _{x \in \mathbb{B}_{R}}\left|\frac{\partial f(x)}{\partial x}\right|+ \\
& \quad R \sup _{x \in \mathbb{B}_{R}}|f(x)| \sup _{x \in \mathbb{B}_{R}}\left|\frac{\partial g(x)}{\partial x}\right|
\end{aligned}
$$

Proof. Omitted.

Thus, using Lemma 2, one obtains a bound on the negative term of (5) through

$$
\begin{aligned}
& \left|\hat{h}_{\mathrm{Si}}^{\prime} \bar{h}_{\mathrm{C}}^{\prime}-\hat{h}_{\mathrm{C}}^{\prime} \bar{h}_{\mathrm{Si}}^{\prime}\right|= \\
& =\mid h_{\mathrm{Si}}^{\prime}(p-\tilde{p}, u) h_{\mathrm{C}}^{\prime}(p-\xi \tilde{p}, u)- \\
& h_{\mathrm{C}}^{\prime}(p-\tilde{p}, u) h_{\mathrm{Si}}^{\prime}(p-\xi \tilde{p}, u) \\
& \leq R \sup _{\tilde{p} \in \mathbb{B}_{R}}\left|h_{\mathrm{Si}}^{\prime}(p-\tilde{p}, u)\right| \sup _{\tilde{p} \in \mathbb{B}_{R}}\left|\frac{\partial h_{\mathrm{C}}^{\prime}(p-\tilde{p}, u)}{\partial \tilde{p}}\right|+ \\
& R \sup _{\tilde{p} \in \mathbb{B}_{R}}\left|h_{\mathrm{C}}^{\prime}(p-\tilde{p}, u)\right| \sup _{\tilde{p} \in \mathbb{B}_{R}}\left|\frac{\partial h_{\mathrm{Si}}^{\prime}(p-\tilde{p}, u)}{\partial \tilde{p}}\right|
\end{aligned}
$$

on the ball $\tilde{p} \in \mathbb{B}_{R}$.

Replacing the first term in (4) and the third term in (5) by their upper bounds and replacing the second term in (5) by its lower bound, yields an upper bound on $\lambda$ which is reasonably tight provided that $\mathbb{B}_{R}$ is small. The bounds in Appendix $\mathrm{B}$, are valid if $R \in \mathbb{I}=\left[0, R_{1}\right)$ where

$$
R_{1} \triangleq \min \left(\frac{v_{1}}{k_{\mathrm{C}}}, \frac{v_{2}}{2 k_{\mathrm{Si}}}\right)
$$

After some rearranging one obtains

$$
\lambda<-N(R) / D(R)
$$

where

$$
\begin{aligned}
N(R)= & 4 a_{1} a_{2}\left(v_{1}+v_{2}-R\left(k_{\mathrm{C}}+2 k_{\mathrm{Si}}\right)\right)^{10} \times \\
& \left(v_{1}+v_{2}+R\left(k_{\mathrm{C}}+2 k_{\mathrm{Si}}\right)^{4}\right) \\
& +4 k\left(v_{1}+v_{2}-R\left(k_{\mathrm{C}}+2 k_{\mathrm{Si}}\right)\right)^{10} \times \\
& \left(4 a_{2} k_{\mathrm{Si}}^{2}\left(v_{1}-k_{\mathrm{C}} R\right)^{4}+a_{1} k_{\mathrm{C}}^{2}\left(v_{2}-2 k_{\mathrm{Si}} R\right)^{4}\right) \\
& -16 R^{2} k^{2} k_{\mathrm{Si}}^{2} k_{\mathrm{C}}^{2}\left(v_{1}+k_{\mathrm{C}} R\right)^{2}\left(v_{2}+2 k_{\mathrm{Si}} R\right)^{2} \times \\
& \left(v_{1}+v_{2}+R\left(k_{\mathrm{C}}+2 k_{\mathrm{Si}}\right)\right)^{6} \times \\
& \left(4 k_{\mathrm{Si}}^{2}\left(v_{1}+k_{\mathrm{C}} R\right)^{2}+k_{\mathrm{C}}^{2}\left(v_{2}+2 k_{\mathrm{Si}} R\right)^{2}\right) \\
D(R)= & 2\left(a_{1}+a_{2}\right)\left(v_{1}+v_{2}-R\left(k_{\mathrm{C}}+2 k_{\mathrm{Si}}\right)\right)^{10} \times \\
& \left(v_{1}+v_{2}+R\left(k_{\mathrm{C}}+2 k_{\mathrm{Si}}\right)\right)^{4}+ \\
& 2 k\left(v_{1}+v_{2}-R\left(k_{\mathrm{C}}+2 k_{\mathrm{Si}}\right)\right)^{6} \times \\
& \left(v_{1}+v_{2}+R\left(k_{\mathrm{C}}+2 k_{\mathrm{Si}}\right)\right)^{4} \times \\
& \left(4 k_{\mathrm{Si}}^{2}\left(v_{1}+k_{\mathrm{C}} R\right)^{2}+k_{\mathrm{C}}^{2}\left(v_{2}+2 k_{\mathrm{Si}} R\right)^{2}\right)
\end{aligned}
$$

The parameters $v_{1}$ and $v_{2}$ are time-dependent and the set inside which every trajectory of $\left[\begin{array}{cc}v_{1} & v_{2}\end{array}\right]^{T}$ is contained is denoted $\mathbb{V}$. Due to process technical reasons 
this region is a subset of a rectangle in the first quadrant.

It is easy to show that there exist $N_{0}, D_{0}, N_{0}^{\prime}$, and $N_{1}^{\prime}$ such that

$$
\begin{aligned}
& 0<N_{0} \leq N(0) \\
& 0<D(R) \leq D_{0} \\
& N_{1}^{\prime} \leq \frac{d N(R)}{d R} \leq N_{0}^{\prime}<0
\end{aligned}
$$

for all $R \in \mathbb{I}$ and $v \in \mathbb{V}$.

Since $N(0)>0$ and $N(R)$ is monotone decreasing on $\mathbb{I}$, the equation

$$
N(R)=0
$$

has one root on this interval or otherwise $N(R)>0$ on $\mathbb{I}$. Let $r$ be this root, if it exists, and otherwise let it be the end of the interval $\mathbb{I}$, i.e. $R_{1}$. Since $N(0)$ and $d N(R) / d R$ are uniformly bounded from below, it is clear that $r$ is uniformly bounded from below by

$$
R_{0} \triangleq \inf _{v \in \mathbb{V}} r \geq-N_{0} / N_{1}^{\prime}>0
$$

An upper bound for $N(R)$ on II can then be expressed as $N(R) \geq\left(R-R_{0}\right) N_{0}^{\prime}$ for all $v \in \mathbb{V}$, which, by (6), implies that $\lambda<-\left(R-R_{0}\right) N_{0}^{\prime} / D_{0}$. Choosing a uniform upper bound $\Lambda_{0}$ for the eigenvalues and solving $-(R-$ $\left.R_{0}\right) N_{0}^{\prime} / D_{0}=\Lambda_{0}$, yields $R=-\Lambda_{0} D_{0} / N_{0}^{\prime}+R_{0}$, which is the radius of a ball inside which $\lambda \leq \Lambda_{0}$.

By letting $\Lambda_{0} \rightarrow 0$, one obtains $R=R_{0}$, i.e. the radius of a ball inside which it is possible to find a uniform negative upper bound for $\lambda$. This implies that $\mathbb{B}_{R_{0}}$ is a region of attraction for $\tilde{p}$. The radius $R_{0}$ of this region is found by solving ( 7 ) for all possible combinations of $v \in \mathbb{V}$ and taking $R_{0}$ to be the smallest of all these solutions. Although $N(R)$ is a polynomial of order 14, this is possible to do with reasonable accuracy, since there is only one root in the interesting interval.

\section{Experimental results}

To find the best value for the feedback gain $k$, estimations of the final value of the carbon content have been performed with different values of $k$. Fig. 5 shows the estimated carbon content at the end of seven heats using (2) with real data from the plant and gain $k=320$. It can be seen that the observer estimate of the carbon content converges to the measured final value of the carbon content (cross).

The results in numbers are presented in Table 1 . The deviation between measured and estimated final carbon content is small compared to e.g. the error window for MEFCON. The larger deviation obtained in one heat is believed to be caused by a large drop in the purge gas

\begin{tabular}{|r|r|r|}
\hline \multicolumn{2}{|c|}{ Final carbon content [\%] } & \\
Measured & Estimated & Error[\%] \\
\hline \hline 0.0830 & 0.0825 & -0.0005 \\
0.0430 & 0.0412 & -0.0018 \\
0.0330 & 0.0339 & 0.0009 \\
0.0260 & 0.0227 & -0.0033 \\
$0 . \dot{\theta} 430$ & 0.0307 & -0.0123 \\
0.0380 & 0.0413 & 0.0033 \\
0.0280 & 0.0310 & 0.0030 \\
\hline
\end{tabular}

Table 1: Comparison between measured and estimated carbon contents for seven heats

flow, which occurred near the end of the blow. Insufficient flow of purge gas results in slower oxidization of carbon. This effect is not accounted for by the model, which explains the underestimation of the carbon content in this heat.

Using (7) to calculate a domain of attraction for $k=$ 320 reveals that $R_{0} \approx 1200$ moles, which is well inside the accuracy of the hot metal analysis.

The output of the observer is an estimate of the decarburization rate (Fig. 2, thick solid line) which is very similar to the estimate obtained from the off-gas analysis (thin solid line), but lacking the large disturbances of the latter.

\section{Conclusions}

It has been shown that by utilizing an analytical model and suitable feedback of the output error, it is possible to obtain accurate estimates of the metal analysis in a top blown converter process. The dynamics of the error when applying this feedback has been shown to be asymptotically stable and a region of attraction has been derived.

\section{Acknowledgements}

The authors want to thank the personnel of SSAB Oxelösund for supplying the data. Financial support provided by the Swedish National Board for Industrial and Technical Development, NUTEK, is also gratefully acknowledged.

\section{References}

[1] http://www.mefos.se/mefcon.htm.

[2] E. A. Misawa and J. K. Hedrick, "Nonlinear observers - a state-of-the-art survey," Journal of Dynamic Systems, Measurment and Control, vol. 111, pp. 344352, September 1989. 

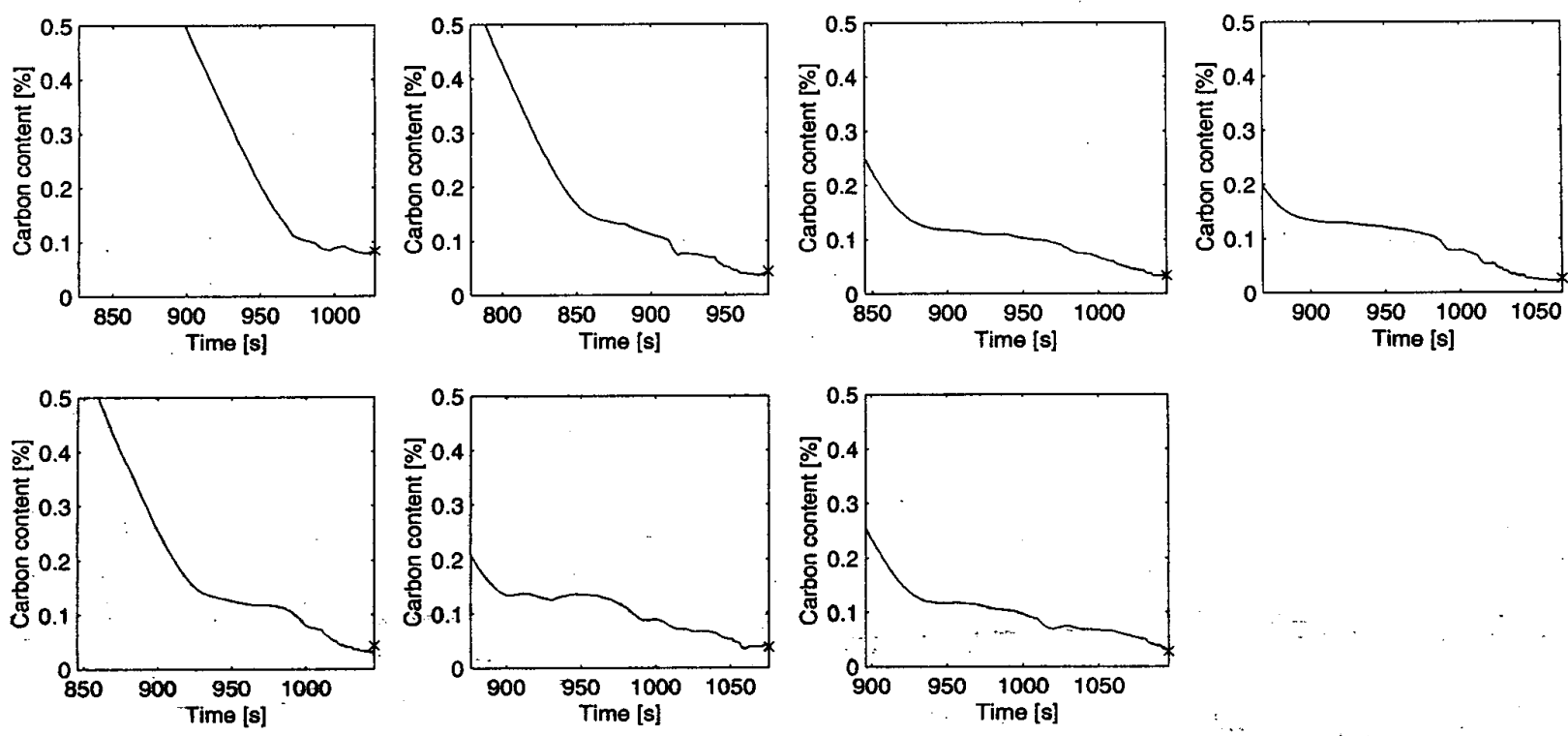

Figure 5: Estimated (line) and measured final value (cross) of carbon content for seven heats.

[3] H. K. Khalil, Nonlinear Systems. Prentice Hall, 2 ed., 1996.

[4] D. Widlund, A. Medvedev, and R. Gyllenram, "Towards model-based closed-loop control of the basic oxygen steelmaking process," in Preprints of the 9th IFAC Symposium Automation in Mining, Mineral and Metal Processing 1998, 1998.

[5] K.-J. Åström and B. Wittenmark, Adaptive Control. Addison-Wesley Publishing Company, Inc., 2 ed., 1995.

\section{A Output transformation}

The off-gas analysis is modeled as

$$
\begin{aligned}
\psi & =\left[\begin{array}{lll}
\psi_{\mathrm{CO}_{2}} & \psi_{\mathrm{CO}} & \psi_{\mathrm{O}_{2}}
\end{array}\right]^{T} \\
& =\left\{\begin{array}{lll}
{\left[\begin{array}{lll}
\frac{2 \eta}{2-\eta} & 0 & \frac{2 \alpha(1-\eta)-\eta}{2-\eta}
\end{array}\right]^{T}} & \eta<\frac{2 \alpha}{1+2 \alpha} \\
{\left[\begin{array}{lll}
\frac{2 \alpha-2 \alpha \eta}{1-\alpha+\alpha \eta} & \frac{2 \alpha(\eta-1)+\eta}{1-\alpha+\alpha \eta} & 0
\end{array}\right]^{T}} & \eta \geq \frac{2 \alpha}{1+2 \alpha}
\end{array}\right.
\end{aligned}
$$

where $\eta=\beta h(p, u) / f_{\text {og }}$.

The decarburization rate is modeled as

$$
h(p, u)=\frac{1}{1 / v_{1}+1 / v_{2}}
$$

where $v_{1}=k_{\mathrm{C}}\left(p_{\mathrm{C}}-p_{\mathrm{C}}^{0}\right)$ and $v_{2}=2\left(u-k_{\mathrm{Si}} p_{\mathrm{Si}}\right)$.

\section{B Sensitivity functions}

The sensitivity function can be expressed as

$$
h^{\prime}(p, u)=\left[\begin{array}{c}
h_{\mathrm{Si}}^{\prime}(p, u) \\
h_{\mathrm{C}}^{\prime}(p, u)
\end{array}\right]=\left[\begin{array}{c}
\frac{-2 k_{\mathrm{Si}} v_{1}^{2}}{\left(v_{1}+v_{2}\right)^{2}} \\
\frac{k_{\mathrm{C}} v_{2}^{2}}{\left(v_{1}+v_{2}\right)^{2}}
\end{array}\right]
$$

The derivatives of the sensitivity functions are

$$
\begin{aligned}
& \frac{\partial h_{\mathrm{Si}}^{\prime}(p, u)}{\partial p}=\left[\begin{array}{c}
\frac{\partial h_{\mathrm{Si}}^{\prime}(p, u)}{\partial p_{\mathrm{Si}}} \\
\frac{\partial h_{\mathrm{Si}}^{\prime}(p, u)}{\partial p_{\mathrm{C}}}
\end{array}\right]=\left[\begin{array}{c}
\frac{-8 k_{\mathrm{Si}}^{2} v_{1}^{2}}{\left(v_{1}+v_{2}\right)^{3}} \\
\frac{-4 k_{\mathrm{Si}} k_{\mathrm{C}} v_{1} v_{2}}{\left(v_{1}+v_{2}\right)^{3}}
\end{array}\right] \\
& \frac{\partial h_{\mathrm{C}}^{\prime}(p, u)}{\partial p}=\left[\begin{array}{c}
\frac{\partial h_{\mathrm{C}}^{\prime}(p, u)}{\partial p_{\mathrm{Si}}} \\
\frac{\partial h_{\mathrm{C}}(p, u)}{\partial p_{\mathrm{C}}}
\end{array}\right]=\left[\begin{array}{c}
\frac{-4 k_{\mathrm{Si}} k_{\mathrm{C}} v_{1} v_{2}}{\left(v_{1}+v_{2}\right)^{3}} \\
\frac{-2 k_{\mathrm{C}}^{2} v_{2}^{2}}{\left(v_{1}+v_{2}\right)^{3}}
\end{array}\right]
\end{aligned}
$$

Approximate upper and lower bounds on the sensitivity functions $h^{\prime}(p-\tilde{p}, u)$ and $h^{\prime}(p-\xi \tilde{p}, u)$ and their derivatives when $\tilde{p} \in \mathbb{B}_{R}, R \in \mathbb{I}$ are obtained as

$$
\begin{aligned}
& \sup _{\tilde{p} \in \mathbb{B}_{R}}\left|h_{\mathrm{Si}}^{\prime}(p-\tilde{p}, u)\right| \geq \frac{2 k_{\mathrm{Si}}\left(v_{1}+k_{\mathrm{C}} R\right)^{2}}{\left(v_{1}+v_{2}-R\left(k_{\mathrm{C}}+2 k_{\mathrm{Si}}\right)\right)^{2}} \\
& \sup _{\tilde{p} \in \mathbb{B}_{R}}\left|h_{\mathrm{C}}^{\prime}(p-\tilde{p}, u)\right| \geq \frac{k_{\mathrm{C}}\left(v_{2}+2 k_{\mathrm{Si}} R\right)^{2}}{\left(v_{1}+v_{2}-R\left(k_{\mathrm{C}}+2 k_{\mathrm{Si}}\right)\right)^{2}} \\
& \inf _{\tilde{p} \in \mathbb{B}_{R}}\left|h_{\mathrm{Si}}^{\prime}(p-\tilde{p}, u)\right| \leq \frac{2 k_{\mathrm{Si}}\left(v_{1}-k_{\mathrm{C}} R\right)^{2}}{\left(v_{1}+v_{2}+R\left(k_{\mathrm{C}}+2 k_{\mathrm{Si}}\right)\right)^{2}} \\
& \inf _{\tilde{p} \in \mathbb{B}_{R}}\left|h_{\mathrm{C}}^{\prime}(p-\tilde{p}, u)\right| \leq \frac{k_{\mathrm{C}}\left(v_{2}-2 k_{\mathrm{Si}} R\right)^{2}}{\left(v_{1}+v_{2}+R\left(k_{\mathrm{C}}+2 k_{\mathrm{Si}}\right)\right)^{2}} \\
& \sup _{\tilde{p} \in \mathbb{B}_{R}}\left|\frac{\partial h_{\mathrm{Si}}^{\prime}(p-\tilde{p}, u)}{\partial \tilde{p}}\right| \geq \frac{4 k_{\mathrm{Si}}\left(v_{1}+k_{\mathrm{C}} R\right) \sqrt{S(R)}}{\left(v_{1}+v_{2}-R\left(k_{\mathrm{C}}+2 k_{\mathrm{Si}}\right)\right)^{3}} \\
& \sup _{\tilde{p} \in \mathbb{B}_{R}}\left|\frac{\partial h_{\mathrm{C}}^{\prime}(p-\tilde{p}, u)}{\partial \tilde{p}}\right| \geq \frac{2 k_{\mathrm{C}}\left(v_{2}+2 k_{\mathrm{Si}} R\right) \sqrt{S(R)}}{\left(v_{1}+v_{2}-R\left(k_{\mathrm{C}}+2 k_{\mathrm{Si}}\right)\right)^{3}}
\end{aligned}
$$$$
\text { where } S(R) \triangleq 4 k_{\mathrm{Si}}^{2}\left(v_{1}+k_{\mathrm{C}} R\right)^{2}+k_{\mathrm{C}}^{2}\left(v_{2}+2 k_{\mathrm{Si}} R\right)^{2} \text {. }
$$ 